\title{
Asymmetrically gauged WZNW models
}

\author{
T. Quella ${ }^{1,2}$ and V. Schomerus ${ }^{2, *}$ \\ ${ }^{1}$ Max-Planck-Institut für Gravitationsphysik, Albert-Einstein-Institut, Am Mühlenberg 1, 14476 Golm, \\ Germany \\ ${ }^{2}$ Service de Physique Théorique, CEA/DSM/SPhT, Unité de recherche associée au CNRS, CEA-Saclay, \\ 91191 Gif sur Yvette Cedex, France
}

Received 31 Januar 2003, accepted 14 March 2003

Published online 27 June 2003

\begin{abstract}
Many interesting exactly solvable backgrounds can be obtained by gauging WZNW models asymmetrically. These include the base of the conifold and the time dependent Nappi-Witten background in which a 3dimensional universe passes through a series of big-bang big-crunch singularities. In this short note we review recent results on the conformal field theory description of asymmetric cosets. In particular, we present formulas for their bulk modular invariant partition functions and for a large number of D-brane boundary states.
\end{abstract}

\section{Introduction}

Cosets $G / H$ form a large class of exactly solvable backgrounds. Usually, $H$ is identified with a subgroup of $G$ and in forming the coset one employs the adjoint action for which $H$ acts symmetrically from the left and from the right. Such symmetric transformations always possess fixed points (e.g. the group unit). These lead to all kinds of singularities of the resulting coset geometry, including boundaries and corners.

It is possible, however, to work with an enlarged class of exactly solvable cosets and this is the theme of the following note. The idea is to admit different left and right actions of $H$ on $G$. Even though conformal invariance imposes strong constraints on asymmetric quotients $G / H$, one gains a lot of freedom in model building. Some of the interesting new theories possess smooth background geometries. One such example is provided by the five-dimensional base $S U(2) \times S U(2) / U(1)$ of the conifold. Other models have isolated singularities such as the big-bang singularity in the four-dimensional Nappi-Witten geometry $S U(2) \times$ $S L(2, \mathbb{R}) / \mathbb{R} \times \mathbb{R}$.

In spite of these interesting features, asymmetric cosets have not been studied very systematically in the past. One reason for this is that they are typically heterotic, i.e. they possess different left and right chiral algebras. Among the few publications which deal with special cases of asymmetric cosets one may find two early publications by Guadagnini et al. [1]. The models which are studied in these papers can be applied to the base of the conifold as was pointed out some years ago by Pando-Zayas and Tseytlin [2]. Actions for a wider class of asymmetrically gauged WZNW models were written down in [3]. We shall recall below that they are relevant for Nappi-Witten type models [4]. The latter have been employed recently to investigate string theory in time-dependent backgrounds with big-bang singularities [5]. Branes in asymmetrically gauged WZNW models were also studied in [6] but our analysis gives boundary theories with a different geometric interpretation.

\footnotetext{
* Corresponding author E-mail: vschomer@spht.saclay.cea.fr
} 


\section{The bulk theory}

In this first section we are going to describe the bulk geometry of asymmetric cosets. To begin with, a detailed formulation of the general setup is given and we review the conditions that conformal invariance imposes on the basic data. We then provide expressions for the bulk partition function.

Two groups $G$ and $H$ enter the construction of a coset $G / H$. Both of them are assumed to be reductive so that they split into a product of simple groups and $U(1)$ factors. Let the number of these factors be $n$ and $r$, respectively, i.e. we take $G$ and $H$ to be of the form $G=G_{1} \times \ldots \times G_{n}$ and $H=H_{1} \times \ldots \times H_{r}$. Furthermore, to each factor $G_{i}$ in the decomposition of $G$ we assign a level $k_{i}$. It is convenient to combine the set of all these levels into a vector $k=\left(k_{1}, \ldots, k_{n}\right)$.

Along with the two groups $G$ and $H$ we need to specify an action of $H$ on $G$. We take the latter to be of the form $g \mapsto \epsilon_{L}(h) g \epsilon_{R}\left(h^{-1}\right)$ where $\epsilon_{L / R}: H \rightarrow G$ denote two group homomorphisms which descend to embeddings of the corresponding Lie algebras. In the usual coset theories $\epsilon_{L}$ and $\epsilon_{R}$ are identical. An asymmetry in the coset construction arises when we drop this condition and allow for two different maps.

The coset space $G / H$ consists of orbits under the action of $H$ on $G$, i.e.

$$
G / H=G /\left[g \sim \epsilon_{L}(h) g \epsilon_{R}\left(h^{-1}\right) ; h \in H\right] .
$$

To be precise, we should display the dependence on the choice of $\epsilon_{L / R}$. But since we consider these maps to be fixed once and for all, we decided to suppress them from our symbol $G / H$ for the coset space. Let us stress, however, that the geometry is very sensitive to the choice of $\epsilon_{L / R}$.

The basic data we have introduced so far, i.e. the two groups $G, H$, the vector $k$ of levels and the maps $\epsilon_{L}$, $\epsilon_{R}$, enter the construction of two-dimensional conformal field theories with target space $G / H$. To ensure conformal invariance, however, these data have to obey one important constraint which we can formulate using the notion of an "embedding index" $x_{\epsilon} \in \operatorname{Mat}(n \times r)$ for the homomorphism $\epsilon: H \rightarrow G$. To define $x_{\epsilon}$ we split $\epsilon$ into a matrix of homomorphisms $\epsilon^{s i}: H_{s} \hookrightarrow G_{i}$ where $s=1, \ldots, r$, and $i=1, \ldots, n$, run through the factors of $H$ and $G$, respectively. The embedding index $x_{\epsilon}=x=\left(x^{s i}\right)$ is a matrix with elements of the form ${ }^{1}$

$$
x^{s i}=\frac{\operatorname{Tr}_{i}\left\{\epsilon^{s i}(X) \epsilon^{s i}(Y)\right\}}{\operatorname{Tr}_{s}\{X Y\}} \text { for } \quad X, Y \in \mathfrak{h}_{s} \backslash\{0\} .
$$

Observe that the number that is computed by the expression on the right hand side does not depend on the choice of the elements $X, Y$. Let us also note that the map $\epsilon^{s i}$ is allowed to map $H_{s}$ onto the unit element in $G_{i}$ for some choices of $i$ and $s$. In this case, the corresponding matrix element $x^{s i}$ vanishes.

Let us now consider the embedding indices $x_{L}$ and $x_{R}$ for the two homomorphisms $\epsilon_{L}$ and $\epsilon_{R}$. A conformal theory with target space $G / H$ exists for our choice of levels $k$, provided that the latter obey the following constraint ${ }^{2}$

$$
x_{L} k=x_{R} k .
$$

In other words, the vector of levels must lie in the kernel of $x_{L}-x_{R}$. For symmetric cosets this condition is trivially satisfied with any choice of $k$. Asymmetric cosets, however, constrain the admissible levels.

Our aim now is to present a few elements of the exact solution. We shall begin with some remarks on the relevant chiral algebras. In the following let us denote the chiral algebra of the WZNW model for the group $G$ and levels $k_{i}$ by $\mathcal{A}(G)$. This algebra is generated by a sum of affine Lie algebras with levels $k_{i}$, one for each factor in the decomposition of the reductive group $G$. The two maps $\epsilon_{L / R}$ give rise to two embeddings of the chiral algebra $\mathcal{A}(\mathfrak{H})$ into $\mathcal{A}(G)$. Let us note that $\mathcal{A}(H)$ is generated by a sum of affine

\footnotetext{
${ }^{1}$ We use a normalized trace $\operatorname{Tr}=2 \operatorname{tr} / I$. Here, we denoted by $\operatorname{tr}$ the matrix trace and by $I$ the Dynkin index of the corresponding representation. We use the conventions of [7, pages 58 and 84].

2 If there are two or more identical groups, this equation has to hold up to a possible relabeling of these groups on one side.
} 
algebras, one for each factor in the product $H=H_{1} \times \ldots \times H_{r}$. The levels of these affine algebras form a vector $\left(k_{s}^{\prime}\right)_{s=1, \ldots, r}$ whose entries are related to the levels of $\mathcal{A}(G)$ by $k^{\prime}=x_{L / R} k$ (matrix notation). Our assumption (2) means that $\epsilon_{L / R}$ give rise to two (possibly different) embeddings of the same chiral algebra $\mathcal{A}(H)$ into $\mathcal{A}(G)$. Given these embeddings, we employ the usual GKO construction to obtain two coset algebras $\mathcal{A}=\mathcal{A}\left(G / H, \epsilon_{L}\right)$ and $\overline{\mathcal{A}}=\mathcal{A}\left(G / H, \epsilon_{R}\right)$ which form the left and right chiral algebras of the asymmetric coset model. Note that these two chiral algebras can be different if the two maps $\epsilon_{L}$ and $\epsilon_{R}$ are not the same. In this sense, asymmetric coset models of the kind that we consider in this note are heterotic conformal field theories.

The state space of an asymmetric coset theory decomposes into representations of the two commuting chiral algebras $\mathcal{A}=\mathcal{A}\left(G / H, \epsilon_{L}\right)$ and $\overline{\mathcal{A}}=\mathcal{A}\left(G / H, \epsilon_{R}\right)$. Our task here is to find a combination of these representations which does not only reflect the geometry of the target space $G / H$ but is at the same time also consistent from a conformal field theory point of view. The second requirement means that the vacuum must be unique and that the partition function is modular invariant.

In the following we label sectors of $\mathcal{A}(G)$ by $\mu, \nu, \ldots$, and we use the letters $a, b, \ldots$, for sectors of $\mathcal{A}(H)$. Let us recall that the two sets of sectors admit an action of the group centers $\mathcal{Z}(G)$ and $\mathcal{Z}(H)$, respectively. This action may be diagonalized by the corresponding modular S-matrices,

$$
S_{J \mu \nu}^{G}=\mathrm{e}^{2 \pi \mathrm{i} Q_{J}(\nu)} S_{\mu \nu}^{G} \quad \text { for } J \in \mathcal{Z}(G)
$$

where $Q_{J}(\nu)=h_{J}+h_{\mu}-h_{J \mu}$ are the so-called monodromy charges. An analogous statement holds for the action of the center $\mathcal{Z}(H)$. In a coset sector $(\mu, a)$ the labels $\mu, a$ form an entity and as such they have to transform identically under the common center (to be specific, we display formulas for the left chiral algebra)

$$
\mathcal{G}_{\text {id }}(L)=\left\{\left(J, J^{\prime}\right) \in \mathcal{Z}(G) \times \mathcal{Z}(H) \mid J=\epsilon_{L}\left(J^{\prime}\right)\right\} .
$$

Not all the labels $(\mu, a)$ fulfill this requirement. What remains is the set

$$
\operatorname{All}(G / H)_{L}=\left\{(\mu, a) \mid Q_{J}(\mu)=Q_{J^{\prime}}(a) \text { for all }\left(J, J^{\prime}\right) \in \mathcal{G}_{\text {id }}(L)\right\}
$$

of allowed coset labels. It turns out that elements in the set $\operatorname{All}(G / H)_{L}$ which are related by the action of $\mathcal{G}_{\text {id }}$ correspond to the same coset sector. The set of sectors for the coset chiral algebra is therefore given by $\operatorname{Rep}(G / H)_{L}=\operatorname{All}(G / H)_{L} / \mathcal{G}_{\text {id }}(L)$. This observation motivates the term "field identification group" for the common center $\mathcal{G}_{\text {id }}(L)$. The same constructions can be performed for the right chiral algebra. But note that in general the resulting expressions will not coincide.

Having introduced all these notions from the representation theory of coset chiral algebras we are finally able to spell out our proposal for the state space,

$$
\mathcal{H}^{G / H}=\bigoplus_{[\mu, a] \in \operatorname{Rep}(G / H)} \mathcal{H}_{(\mu, a)}^{(G / H)_{L}} \otimes \overline{\mathcal{H}}_{(\mu, a)^{+}}^{(G / H)_{R}} .
$$

where the set $\operatorname{Rep}(G / H)$ is defined by

$$
\begin{aligned}
& \operatorname{Rep}(G / H)=\operatorname{All}(G / H) / \mathcal{G}_{\text {id }} \quad \text { with } \\
& \operatorname{All}(G / H)=\operatorname{All}(G / H)_{L} \cap \operatorname{All}(G / H)_{R}, \quad \mathcal{G}_{\text {id }}=\mathcal{G}_{\text {id }}(L) \cap \mathcal{G}_{\text {id }}(R) .
\end{aligned}
$$

Note that the field identification group $\mathcal{G}_{\text {id }}$ admits a natural interpretation as the stabilizer of the action $g \mapsto \epsilon_{L}(h) g \epsilon_{R}(h)^{-1}$, i.e.

$$
\mathcal{G}_{\mathrm{id}}=\left\{\left(J, J^{\prime}\right) \mid J^{\prime} \in \mathcal{Z}(H), J=\epsilon_{L}\left(J^{\prime}\right)=\epsilon_{R}\left(J^{\prime}\right) \in \mathcal{Z}(G)\right\} .
$$


In writing our formula (3) we implicitly assumed that the action of the field identification group $\mathcal{G}_{\text {id }}$ on $\operatorname{All}(G / H)$ possesses no fixed points, i.e. that all orbits $[\mu, a]$ have the same length. It should be stressed that fixed points for the action of $\mathcal{G}_{\text {id }}(L / R)$ on $\operatorname{All}(G / H)_{L / R}$ are not ruled out by this assumption. It has been shown in [8] that (3) is modular invariant and that it contains the vacuum sector with multiplicity one. Furthermore, the subspace of ground states for which the conformal dimension vanishes when the levels are sent to infinity has been shown to agree with the space of functions on the geometric coset $G / H$.

\section{The boundary theory}

In this section we discuss the boundary states for asymmetric coset theories that have been found in [8]. Since these are obtained from symmetry breaking branes on $G$, our discussion starts with a short review on branes in group manifolds. We then argue that some of the symmetry breaking branes on $G$ can descend to the asymmetric coset and present formulas for their boundary states.

Among the branes on group manifolds, maximally symmetric branes are distinguished since they preserve the whole chiral current algebra symmetry. The construction of maximally symmetric boundary conditions in the WZNW model requires to choose some gluing automorphism $\Omega$ of the chiral algebra $\mathcal{A}(G)$ so that we can glue holomorphic and anti-holomorphic currents along the boundary. Before we describe a few results from boundary conformal field theory of the corresponding branes, let us briefly look at the geometric scenario these boundary conditions are associated with. It is by now well known that branes constructed with $\Omega=$ id are localized along conjugacy classes [9]. The general case has an equally simple and elegant interpretation [10]. Note that gluing automorphisms $\Omega$ for the current algebra $\mathcal{A}(G)$ are associated with automorphisms of the finite dimensional Lie algebra $\mathfrak{g}$ which, after exponentiation, give rise to an automorphism $\Omega^{G}$ of the group $G$. One can then show that maximally symmetric branes are localized along the following twisted conjugacy classes in the group manifold,

$$
\mathcal{C}_{u}^{\Omega}:=\left\{g u \Omega^{G}\left(g^{-1}\right) \mid g \in G\right\} .
$$

The subsets $\mathcal{C}_{u}^{\Omega} \subset G$ are parametrized through equivalence classes of group elements $u$ where the equivalence relation between two elements $u, v \in G$ is given by: $u \sim_{\Omega} v$ iff $v \in C_{u}^{\Omega}$. One should think of $u$ as a coordinate that describes the transverse position of the brane on the group manifold. In the exact conformal field theory, these coordinates are quantized.

The algebraic description of maximally symmetric D-branes was developed in [11] (see also [12]). Their boundary states are labeled by representations of the twisted Kac-Moody algebra which may be constructed from the Lie algebra $\mathfrak{g}$ using the automorphism $\Omega$. They are specific linear combinations of certain generalized coherent (or Ishibashi) states,

$$
\left.|u\rangle=\sum_{\Omega(\mu)=\mu} \frac{\psi_{u}^{\mu}}{\sqrt{S_{0 \mu}}}|\mu\rangle\right\rangle .
$$

As usual, the generalized coherent states only implement the gluing conditions for the currents and there is one such state for each $\Omega$-symmetric combination of irreducible $\hat{\mathfrak{g}}$-representations in the charge conjugate state space of the WZNW theory. The coefficients $\psi_{u}{ }^{\mu}$ in the previous formula are directly related to the one-point functions of bulk fields in the boundary theories and explicit expressions can be found in the literature [11]. From the boundary states one can compute the partition function

$$
Z_{u v}=\sum_{\nu \in \operatorname{Rep}(G)}\left(n_{\mu}\right)_{v}{ }^{u} \chi_{\nu}=\sum_{\nu \in \operatorname{Rep}(G)} \sum_{\mu=\Omega(\mu)} \frac{\bar{\psi}_{u}^{\mu} \psi_{v}{ }^{\mu} S_{\nu \mu}}{S_{0 \mu}} \chi_{\nu}
$$

for each pair of labels $u, v$. The numbers $\left(n_{\mu}\right)_{v}{ }^{u} \in \mathbb{N}_{0}$ are the twisted fusion rules of $\hat{\mathfrak{g}}$. For details of the construction we refer the reader to the existing literature. 
In addition to these maximally symmetric branes, a large class of symmetry breaking branes has been obtained in [13]. Their geometry was identified later in [14]. The construction of these branes requires to choose a chain of groups $U_{s}, s=1, \ldots, N$, along with homomorphisms $\epsilon_{s}: U_{s} \rightarrow U_{s+1}$ (we set $U_{N}=G$ ). The latter are again assumed to induce embeddings of the corresponding Lie algebras. Furthermore, one has to select an automorphism $\Omega_{s}$ on each group $U_{s}$. Given these data, it is possible to construct a set of branes which preserve an $U_{1}$ group symmetry. These are localized along the following sets

$$
\begin{aligned}
\mathcal{C}_{\underline{\underline{\epsilon}} ; \underline{u}}^{\Omega} & =\mathcal{C}_{u_{N}}^{N} \cdot \mathcal{C}_{u_{N-1}}^{N-1} \cdot \ldots \cdot \mathcal{C}_{u_{1}}^{1} \subset G \text { where } \\
\mathcal{C}_{u_{s}}^{s} & =\Omega_{N} \circ \epsilon_{N-1} \circ \ldots \circ \Omega_{s+1} \circ \epsilon_{s}\left(\mathcal{C}_{u_{s}}^{\Omega_{s}}\right) \subset G \text { for } u_{s} \in U_{s}
\end{aligned}
$$

and $\mathcal{C}_{u_{N}}^{N}=\mathcal{C}_{u_{N}}^{\Omega_{N}}$ for $u_{N} \in G$. The $\cdot$ indicates that we consider the set of all points in $G$ which can be written as products (with group multiplication) of elements from the various subsets. One should stress that branes may be folded onto the subsets (6) such that a given point is covered several times. This phenomenon has been observed for a special case in [15].

Having constructed maximally symmetric and symmetry breaking branes in the group $G$, our strategy now is to investigate which of these branes can pass down to the asymmetric coset $G / H$. Geometrically, this is not too hard to understand. In fact, the natural idea is to look at all the symmetry breaking branes which are obtained from chains starting with $U_{1}=H$ and end at $U_{N}=G$ and to impose an extra condition on the choice of the automorphisms $\Omega_{s}$ and the homomorphisms $\epsilon_{s}$ so as to reflect the action of $H$ on $G$ in the coset construction. Explicitly, the conditions on $\Omega_{s}$ and $\epsilon_{s}$ read

$$
\epsilon_{L}=\epsilon_{N-1} \circ \ldots \circ \epsilon_{1}
$$

and

$$
\epsilon_{R}=\Omega^{U_{N}} \circ \epsilon_{N-1} \circ \Omega^{U_{N-1}} \circ \ldots \circ \Omega^{U_{2}} \circ \epsilon_{1} \circ \Omega^{U_{1}}
$$

Our claim is that the subsets (6) that are obtained from chains $\left(U_{s}, \Omega_{s}\right)$ with homomorphisms $\epsilon_{s}$ pass down to subsets on the asymmetric coset $G / H$, provided that the data of the chain are related to the data $\epsilon_{L / R}$ of the asymmetric coset $G / H$ by eqs. (7) and (8).

It turns out that these geometric ideas are indeed correct and that there exist exact boundary conformal field theories which describe the branes wrapping the subsets (6), (7) and (8). Our assumptions on the existence of a chain of embeddings and its properties guarantee that the resulting theories are rational with respect to a reduced chiral symmetry

$$
\mathcal{A}=\mathcal{A}\left(U_{N} / U_{N-1}\right) \otimes \ldots \otimes \mathcal{A}\left(U_{3} / U_{2}\right) \otimes \mathcal{A}\left(U_{2} / U_{1}\right)
$$

For the explicit formulas, let us now restrict to embedding chains of length $N=3$. This does not only cover the most interesting examples, but it also simplifies our notations. The extension to the general case is straightforward. We will also set $\Omega_{1}=\mathrm{id}=\Omega_{3}$ and write $U_{2}=U$.

Before we spell out our boundary states it is convenient to rewrite the bulk partition function of the asymmetric coset model in terms of characters for the chiral algebra $\mathcal{A}$ that is left unbroken by the boundary condition. With our simplifying assumptions, this partition function becomes

$$
\mathcal{H}=\bigoplus_{[\mu, a] \in \operatorname{Rep}(G / H)} \bigoplus_{\beta \in \operatorname{Rep}(U)} \mathcal{H}_{(\mu, \alpha)}^{G / U} \otimes \mathcal{H}_{(\alpha, a)}^{U / H} \otimes \overline{\mathcal{H}}_{(\mu, \beta)^{+}}^{G / U} \otimes \overline{\mathcal{H}}_{(\Omega(\beta), a)^{+}}^{U / H} .
$$

We had to include the automorphisms $\Omega$ in one of the coset representations because in the original formulation of the symmetry reduction left and right chiral algebra are just isomorphic, not identical. By explicit insertion of $\Omega$ we are able to formulate the theory in terms of one single chiral algebra $\mathcal{A}$. 
To construct boundary states we have to find the symmetric part of the Hilbert space (9). From the $G / U$ cosets we obtain the condition $\alpha \equiv \beta$ modulo field identification of the form $(0, J) \in \mathcal{G}_{\text {id }}(G / U)$. From the $U / H$ cosets one arrives at $\alpha=\Omega(\beta)$. This is due to the fact that elements of the field identification group $\mathcal{G}_{\text {id }}(U / H)$ can not have the form $(J, 0)$. The first condition then translates into $\alpha=J \Omega(\alpha)$. We will assume that this condition can only be fulfilled for $\Omega(\alpha)=\alpha \cdot{ }^{3}$ Generalized coherent states $\left.|\mu, \alpha, a\rangle\right\rangle$ for this setup are labeled by triples $\mu, \alpha, a$ such that

$$
\begin{gathered}
(\mu, \alpha) \in \operatorname{All}(G / U), \quad(\alpha, a) \in \operatorname{All}(U / H), \\
\Omega(\alpha)=\alpha .
\end{gathered}
$$

In addition we have to identify these generalized coherent states according to the identification rule

$$
\left.\left.\left|J \mu, \alpha, J^{\prime} a\right\rangle\right\rangle \sim|\mu, \alpha, a\rangle\right\rangle \text { for }\left(J, J^{\prime}\right) \in \mathcal{G}_{\text {id }} .
$$

Let $\psi_{z}{ }^{\alpha}$ be the structure constants of twisted D-branes in the target space $U$. Then we may define boundary states for the asymmetric coset by

$$
\left.|\rho, z, r\rangle=\sum \frac{S_{\rho \mu}^{G}}{\sqrt{S_{0 \mu}^{G}}} \frac{\psi_{z}^{\alpha}}{S_{0 \alpha}^{U}} \frac{\bar{S}_{r a}^{H}}{\sqrt{S_{0 a}^{H}}}|\mu, \alpha, a\rangle\right\rangle .
$$

We note that this is a consistent prescription since the formula does not depend on the specific representative of the Ishibashi states. Using world-sheet duality it is not difficult to derive formulas for the boundary partition functions,

$$
Z=\sum N_{\rho_{1}^{+}, \rho_{2}, \nu} N_{\beta^{+} \gamma}^{\delta}\left(n_{\delta}\right)_{z_{1}}^{z_{2}} N_{r_{1} r_{2}^{+}}^{b} \chi_{(\nu, \beta)}^{G / U} \chi_{(\gamma, b)}^{U / H}(q)
$$

Here, $n$ are the multiplicities that appear in the spectrum of twisted branes on group manifolds. Let us remark that the spectrum (10) is consistent with the proposed geometric interpretation.

The abstract formulas we presented in this note can be illustrated through a number of important examples. Among them are the spaces $T^{p q}=S U(2) \times S U(2) / U(1)$. Here, the integers $p, q$ label different embeddings of the denominator group $U(1)$. For $p=1, q=1$ one obtains a close relative of the base of the conifold in which the RR-fluxes of the latter are replaced by a NSNS background field [2]. Our general theory provides a large class of boundary theories for this background, including branes that wrap one of the 3-spheres in $T^{11}$. Related objects play an important role in the conifold geometry.

Recently, there has been renewed interest [5] in the Nappi-Witten background [4] which describes a closed universe between a big-bang and a big-crunch singularity. It was shown that the dynamics couples the closed universe to regions in space-time which formerly were believed to be unphysical. The full geometry is given by the coset $S L(2, \mathbb{R}) \times S U(2) / \mathbb{R} \times \mathbb{R}$ where the groups in the numerator act asymmetrically on both factors in the denominator. Once more, our general geometric picture can be applied and provides a large set of possible brane geometries. We believe that the construction of the corresponding boundary states in these non-compact backgrounds is possible using results from $[16,17]$.

Acknowledgements We would like to thank the organizers of the symposium for an inspiring and enjoyable meeting.

\footnotetext{
3 This condition can be non-trivial only if there exist elements in the center of $H$ which are mapped to the unit element by both $\epsilon_{L}$ and $\epsilon_{R}$.
} 


\section{References}

[1] E. Guadagnini, M. Martellini, and M. Mintchev, Scale invariant sigma models on homogeneous spaces, Phys. Lett. B 194, 69 (1987).

E. Guadagnini, Current algebra in sigma models on homogeneous spaces, Nucl. Phys. B 290, 417 (1987).

[2] L. A. Pando-Zayas and A. A. Tseytlin, Conformal sigma models for a class of T(p,q) spaces, Class. Quantum Gravity 17, 5125-5131 (2000) [hep-th/ 0007086 ].

[3] I. Bars and K. Sfetsos, Generalized duality and singular strings in higher dimensions, Mod. Phys. Lett. A 7, 1091-1104 (1992) [hep-th/9110054].

[4] C. R. Nappi and E. Witten, A closed, expanding universe in string theory, Phys. Lett. B 293, 309-314 (1992) [hep-th/9206078].

[5] S. Elitzur, A. Giveon, D. Kutasov, and E. Rabinovici, From big bang to big crunch and beyond, JHEP 06, 017 (2002) [hep-th/0204189].

[6] M. A. Walton and J.-G. Zhou, D-branes in asymmetrically gauged WZW models and axial-vector duality, hep-th/ 0205161.

[7] J. Fuchs, Affine Lie algebras and quantum groups, Cambridge Monographs on Mathematical Physics (Cambridge University Press, Cambridge, 1995).

[8] T. Quella and V. Schomerus, Asymmetric cosets, hep-th/ 0212119.

[9] A. Yu. Alekseev and V. Schomerus, D-branes in the WZW model, Phys. Rev. D 60, 061901 (1999) [hep-th/9812193].

[10] G. Felder, J. Fröhlich, J. Fuchs, and C. Schweigert, The geometry of WZW branes, J. Geom. Phys. 34, 162-190 (2000) [hep-th/9909030].

[11] L. Birke, J. Fuchs, and C. Schweigert, Symmetry breaking boundary conditions and WZW orbifolds, Adv. Theor. Math. Phys. 3, 671-726 (1999) [hep-th/ 9905038 ].

[12] R. E. Behrend, P. A. Pearce, V. B. Petkova, and J.-B. Zuber, Boundary conditions in rational conformal field theories, Nucl. Phys. B 570, 525-589 (2000) [hep-th/ 9908036 ].

[13] T. Quella and V. Schomerus, Symmetry breaking boundary states and defect lines, JHEP 06, 028 (2002) [hep-th/0203161].

[14] T. Quella, On the hierarchy of symmetry breaking D-branes in group manifolds, JHEP 12, 009 (2002) [hep-th/0209157].

[15] J. Maldacena, G. W. Moore, and N. Seiberg, Geometrical interpretation of D-branes in gauged WZW models, JHEP 07, 046 (2001) [hep-th / 0105038$].$

[16] B. Ponsot, V. Schomerus, and J. Teschner, Branes in the euclidean AdS(3), JHEP 02, 016 (2002) [hep-th/0112198].

[17] P. Lee, H. Ooguri, and J.-w. Park, Boundary states for AdS(2) branes in AdS(3), Nucl. Phys. B 632, 283-302 (2002) [hep-th/0112188]. 